\title{
Raman Phonon Spectra of Isotopic Mixed Naphthalene Crystals: Librational Exciton Model and the Amalgamation Limit*
}

\author{
Paras N. Prasad and Raoul Kopelman \\ Department of Chemistry, The University of Michigan, Ann Arbor, Michigan 48104
}

(Received 13 March 1972)

\begin{abstract}
We present an experimental Raman study of lattice modes in neat and mixed $(10 \%-90 \%)$ crystals of naphthalene- $h_{8}$ and naphthalene- $d_{8}$ at $100^{\circ} \mathrm{K}$ with $1 \mathrm{~cm}^{-1}$ resolution. The spectral features of the neat crystals are preserved in the heavily doped mixed crystals, with small shifts and broadenings characteristic of an amalgamation limit that assumes weakly coupled excitation bands in the restricted Frenkel-Davydov limit. Rotation-translation interaction does not affect the mixed crystal spectra, thus making the Raman technique uniquely suited for the investigation of the librational (rotational) phonon band structure. The evidence is against localized or pseudolocalized phonons in these isotopic mixed crystals.
\end{abstract}

\section{INTRODUCTION}

Vibrational phonons arising from intramolecular normal modes in molecular crystals have been successfully treated as vibrational excitons. ${ }^{1-5}$ The best understood Frenkel-Davydov excitons are actually vibronic excitons. ${ }^{5,6}$ The question arises whether the concept of librational (rotational) exciton is useful. The study of phonon states in mixed crystals may provide an answer to it. The exciton states in mixed molecular solids have been widely investigated and reasonably good theoretical recipes exist ${ }^{7-13}$ for dealing with such excitations in isotopically mixed crystals (both dilute and heavily doped). The phonon states in mixed molecular solids, on the other hand, have not received much attention. ${ }^{14,15}$ The nature of phonon bands even in the neat organic crystals is not well understood. ${ }^{16}$ Under these circumstances it will be helpful to find out whether the librational exciton is a useful concept.

In this paper we present an experimental Raman study of isotopic mixed crystals which is fully consistent with an amalgamation limit and supports, if not requires, the concept of librational excitons in both neat and mixed molecular crystals. A detailed discussion on the validity of such a concept and the comparative study of the phonon approach and the exciton approach will be presented in a future publication. ${ }^{17}$ The system chosen here was naphthalene, for the obvious reason that the naphthalene crystalline state has been widely investigated..$^{18-22}$

The mixed crystal studies also provide us with information regarding the nature of phonon bands, the validity of the separation between librational and translational motions, line broadening, band mixing, and localization vs delocalization in the mixed crystals. A study on the vibrational exciton bands of mixed naphthalene crystals is given in the preceding paper.

\section{EXPERIMENTAL}

Naphthalene- $h_{8}$ and naphthalene- $d_{8}$ were purified by repeated zone refining after potassium fusion. Neat crystals and mixed crystals of various concentration mixtures were grown from the melt in a capillary under vacuum. The capillary containing the crystal (which remained under vacuum) was used for the experiment. It was cooled by a flow of cold nitrogen vapor which provided an effective bath of about $100^{\circ} \mathrm{K}$. The spectra were recorded photoelectrically using direct current on a Ramalog-Spex double spectrometer model 1401. An argon ion laser from Coherent Radiation Laboratory was used as the excitation source. The excitation line was the $4880 \AA$ laser emission which was selected by a suitable interference filter. The spectral resolution was about $1 \mathrm{~cm}^{-1}$. The naphthalene- $d_{8}$ was from ICN (International Chemical and Nuclear Corporation, Irvine, California), $99.5 \%$ atom deuterium. The mixed crystals investigated had concentrations of about $10 \%, 30 \%, 50 \%, 70 \%$, and $90 \%$ naphthalene- $d_{8}$.

\section{RESULTS AND DISCUSSION}

The results are shown in Fig. 1. With the exception of slight frequency differences, our result on neat crystals is in agreement with that of Ito et al. ${ }^{22}$ The spectrum consists of six $\mathbf{k}=0$ modes. They belong to three subbands of different librational motions with each of the subbands consisting of two "Davydov" branches. The two components of the Davydov branches have the symmetries $B_{a}$ and $A_{g}$. The frequencies of these six modes are: $56.5\left(B_{g}\right), 67\left(A_{g}\right) ; 83\left(B_{q}\right), 87\left(A_{q}\right)$; $120\left(A_{q}\right), 140\left(B_{q}\right)$ for naphthalene- $h_{8}$; and $51\left(B_{q}\right)$, $63\left(A_{q}\right) ; 78\left(B_{g}\right), 83\left(A_{g}\right) ; 111\left(A_{q}\right), 129\left(B_{g}\right) \mathrm{cm}^{-1}$ for naphthalene- $d_{8}$, at about $100^{\circ} \mathrm{K}$. We have looked carefully for additional bands in mixed crystals (especially of lower concentration, $\sim 10 \%$ ) in the region 10 to $200 \mathrm{~cm}^{-1}$. There does not seem to be any new band appearing in going from the neat crystal to the mixed crystal..$^{23}$ This suggests the absence of localized and pseudolocalized modes. ${ }^{24-26}$

The modes in the region 50 to $90 \mathrm{~cm}^{-1}$ appear unchanged in shape and show only a little change in linewidth in going from the neat crystal to the mixed crystal. Considering that these modes are embedded in the translational phonon continuum with a large density 


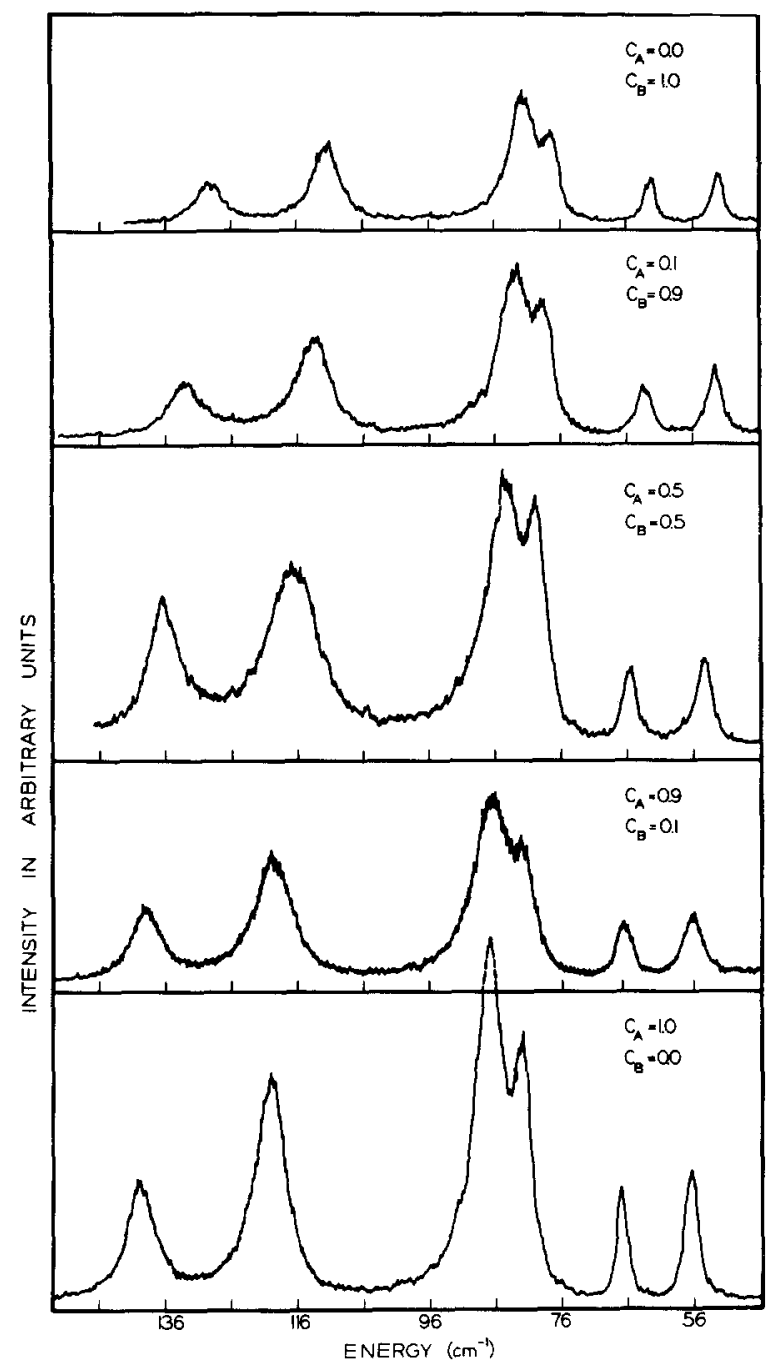

FIG. 1. Raman Spectra of neat and mixed crystals of naphthalene- $h_{8}(A)$ and naphthalene- $d_{8}(B)$ at a spectral slitwidth of about $1 \mathrm{~cm}^{-1}$ and a temperature of about $100^{\circ} \mathrm{K}$, for five different concentrations $(C)$.

of phonon states in this region, ${ }^{19,20}$ we suggest that, within the limits of our sensitivity, there is no significant mixing between the translational modes and the librational modes. In other words, the mixed crystal system, to a reasonable degree of approximation, preserves the site symmetry $\mathbf{C}_{i}$. Furthermore, this suggests little mixing of the rotational phonon subbands (vide infra).

Another important feature to be noticed is the frequency shift of every mode in going from one component of the mixed crystal system to another. This shift is practically linear in concentration (Fig. 2), thus showing that the isotopic mixed crystal system approaches the amalgamation limit ${ }^{10,27,28}$ where the trap depth for each subband is much smaller than the bandwidth of the neat crystal subband. The above is further evidence against localized phonons. ${ }^{26}$
The intensity ratio of the two Davydov components $\left(A_{\theta}\right.$ and $B_{\theta}$ ) in any subband of the isotopic mixed crystals mimics the behavior of the pure crystals. This suggests that the impurity does not introduce appreciable mixing between the two branches in any subband. In other words, the librational phonon (or librational exciton, as we would like to call it) satisfies the restricted Frenkel ${ }^{30}$ limit in which the dominant role is played by short-range interactions. In this limit the self-energy term is approximately diagonal in the branch index. ${ }^{13}$

Finally, we would like to comment on the line broadening observed in the mixed crystals. The most significant broadening is observed in the modes of 120 and $140 \mathrm{~cm}^{-1}$, of napthhalene- $h_{8}$, in mixed crystals. The maximum broadening is seen in a $50 \%$ mixture. The half-widths of these modes in a neat naphthalene- $h_{8}$ crystal are 5.5 and $5 \mathrm{~cm}^{-1}$, whereas in a $50 \%$ mixture they are 10 and $7 \mathrm{~cm}^{-1}$, respectively. This broadening is not a result of mixing with translational phonons, as the highest translational phonon frequency is only about $105 \mathrm{~cm}^{-1}$ (at liquid nitrogen temperature). ${ }^{31}$ The broadening can be partially explained by a calculation based on a moment expansion at the amalgamation limit. ${ }^{28}$ According to the result of this calculation, the broadening of different modes in a mixed crystal

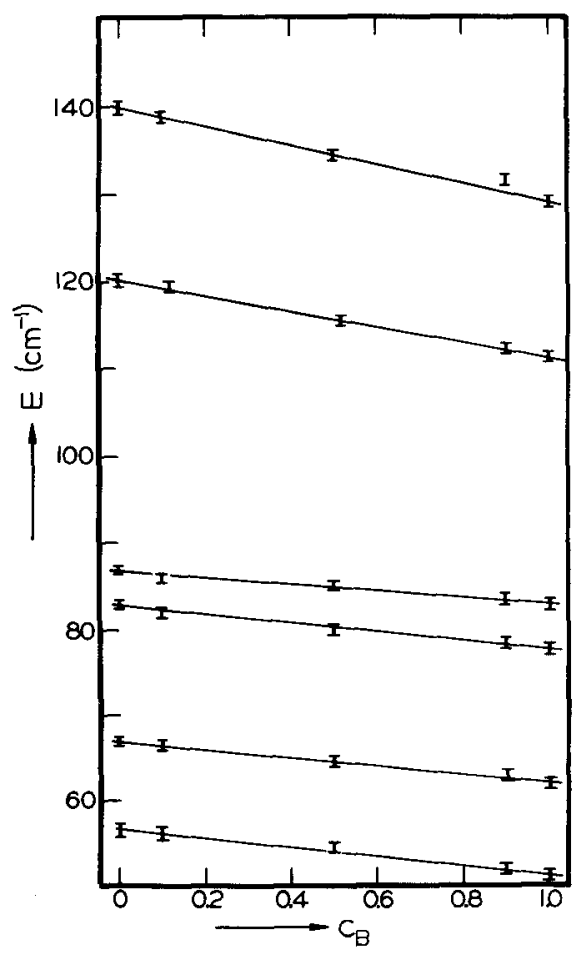

FIG. 2. Concentration dependence of Raman active lattice mode energies of naphthalene- $h_{8}(A)$-Naphthalene- $d_{8}(B)$ mixed crystals. Note that this figure does not indicate line broadenings, only peak positions and their uncertainties. 
of fixed composition is proportional to the trap depth and the broadening is largest in a $50 \%$ mixture. Our result is in agreement with such predictions. The trap depth corresponding to the 120 and $140 \mathrm{~cm}^{-1}$ mode is $10 \mathrm{~cm}^{-1}$, whereas the trap depths for others are around $5 \mathrm{~cm}^{-1}$. However, considering the fact that the two modes (120 and $140 \mathrm{~cm}^{-1}$ ) are two Davydov components, one would expect them to be equally broadened in mixed crystals. We suggest that the additional broadening in the $120 \mathrm{~cm}^{-1}$ mode might be because of the fact that it lies just above another mode of the same symmetry $\left(A_{0}\right)$ with which it can mix under the added perturbation of the mixed crystal. Such a situation does not arise with other modes, where usually a $B_{g}$ mode lies just above an $A_{o}$ mode and vice versa.

\section{CONCLUSION}

Our mixed crystal studies show that the six rotational zone-center modes observed for neat crystals of naphthalene can be considered as three pairs of Davydov (factor group, interchange group) components, corresponding to three distinct rotational "exciton" bands with little interband mixing and rotationtranslation (libration-vibration) mixing, and even little intraband mixing in the mixed crystal. This behavior is typical of restricted Davydov-Frenkel excitons. In addition, there is evidence against localized or pseudolocalized phonon modes in any of these isotopic mixed crystals. The Raman spectra of isotopic mixed crystals are thus found to be an ideal tool for the investigation of librational (rotational) phonons in molecular crystals. Note added in proof: The evidence given of the amalgamation limit serves as a basis for our new method of mapping the complete phonon-density of states [R. Kopelman, F. W. Ochs and P. N. Prasad (unpublished) ].

\section{ACKNOWLEDGMENT}

We thank the National Science Foundation for grant GP-8345, which enabled the departmental purchase of the Ramalog-Spex double spectrometer.

* Supported by NSF Grant GP-18718 and NIH Grant NS08116. ${ }^{1}$ E. R. Bernstein and G. W. Robinson, J. Chem. Phys. 49, $4962(1968)$

${ }^{2}$ A. R. Gee and G. W. Robinson, J. Chem. Phys. 46, 4847 (1967)
${ }^{3}$ R. Kopelman, J. Chem. Phys. 47, 3227 (1967); J. C. Laufer and R. Kopelman, ibid. 53, 3674 (1970).

${ }^{4}$ R. M. Hexter, J. Chem. Phys. 33, 1833 (1960); 36, 2285 (1962).

${ }_{5}^{5}$ G. W. Robinson, Ann. Rev. Phys. Chem. 21, 429 (1970).

${ }^{6}$ H. K. Hong and R. Kopelman, J. Chem. Phys. 55, 724 (1971).

7 Y. A. Izyumov, Advan. Phys. 14, 569 (1965).

${ }^{8}$ B. S. Sommer and J. Jortner, J. Chem. Phys. 50, 187 (1969); 50, $822(1969)$.

${ }^{9} \mathrm{~F}$. Yonezawa and T. Matsubara, Progr. Theoret. Phys. (Kyoto) 35, 357 (1966) ; 35, 759 (1966) ; 37, 1346 (1967).

${ }_{10} \mathrm{Y}$. Onodera and Y. Toyozawa, J. Phys. Soc. Japan 24, 341 (1968).

${ }^{11}$ H. K. Hong and G. W. Robinson, J. Chem. Phys. 52, 825 (1970) ; J. Chem. Phys. 54, 1369 (1971).

${ }_{12} \mathrm{~J}$. Hoshen and J. Jortner, J. Chem. Phys. 56, 933 (1972).

${ }_{13} \mathrm{H}$. K. Hong and R. Kopelman, J. Chem. Phys. 55, 3491 (1971) ; 55, 5380 (1971).

${ }_{14}$ G. Venkataraman and V. C. Sahni, Rev. Mod. Phys. 24, 409 (1970).

${ }^{25}$ D. W. Taylor, Phys. Rev. 156, 1017 (1967).

${ }_{16}$ A. B. Zahlan (ed.), Excitons, Phonons and Magnons in Molecular Crystals (Cambridge U. P., Cambridge, England, 1968).

17 P. N. Prasad and R. Kopelman (unpublished).

18 D. W. J. Cruickshank, Acta Cryst. 9, 754 (1956); Rev. Mod. Phys. 30, 163 (1958).

${ }_{19}$ G. S. Pawley, Phys. Status Solidi 20, 347 (1967).

${ }^{20}$ G. S. Pawley and S. J. Cyvin, J. Chem. Phys. 52, 4073 (1970)

${ }^{21}$ B. S. Pawley and E. A. Yeats, Solid State Commun. 7, 385 (1969).

${ }_{22}$ M. Ito, M. Suzuki and T. Yokoyama, in Ref. 16, p. 1.

${ }^{23}$ Our naphthalene- $d_{8}$ does not show a band at about 160 $\mathrm{cm}^{-1}$ (not even at room temperature), in contrast to that reported by A. Bree and R. A. Kydd, Spectrochim. Acta 26a, 1791 (1970). We do, sometimes, see some weak bands at about $40 \mathrm{~cm}^{-1}$ in both neat and mixed crystals. These, we feel, are related to the pure crystal phonon density-of-states peaks and will be discussed further in the future. [R. Kopelman, F. W. Ochs and P. N. Prasad, (unpublished).]

${ }^{24}$ P. H. Chereson, P. S. Friedman, and R. Kopelman, J. Chem. Phys. 56, 3716 (1972).

${ }^{25}$ R. M. Hochstrasser and P. N. Prasad, J. Chem. Phys. 56, 2814 (1972).

${ }_{26}$ In contrast to some suggestions by D. M. Hanson, J. Chem. Phys. 51, 5063 (1969). We feel now that these data should be interpreted in terms of the host phonon density-of-states peaks [R. Kopelman, F. W. Ochs and P. N. Prasad, (unpublished)], in agreement with the present views of D. M. Hanson (private communication). Compare also G. Dolling and B. M. Powell, Proc. Roy. Soc. (London) A319, 209 (1970).

${ }^{27}$ H. Matsuda and T. Miyota, Prog. Theor. Phys. Suppl. 450 (1968).

${ }^{28} \mathrm{~J}$. Hoshen, Ph.D. thesis, Tel-Aviv University, 1971; also Ref. 12 .

${ }^{29}$ The trap depth represents the difference for any subband between the naphthalene- $h_{8}$ mean frequency and the corresponding naphthalene- $d_{8}$ mean frequency.

${ }^{30}$ S. D. Colson, R. Kopelman, and G. W. Robinson, J. Chem. Phys. 47, 27, 5462 (1967).

${ }_{31}$ A. Hadni, B. Wyncke, G. Morlot, and X. Gerbaux, J. Chem. Phys. 51, 3514 (1969). 